\title{
INATIVAÇÃO DE PSEUDOMONAS AERUGINOSA EM RESíDUOS DE SERVIÇOS DE SAÚDE POR MICROONDAS
}

\author{
MicRoWAVE INACTIVATION OF PSEUdOMONAS AERUGINOSA IN \\ HEALTHCARE WASTE
}

\begin{abstract}
REINALDO PISANI JÚNIOR
Engenheiro Químico pela Universidade Federal de São Carlos. Mestre em Engenharia Química pela Universidade Federal de São Carlos. Doutor em Engenharia Química pela Universidade Federal de São Carlos. Docente do Programa de Pós-

Graduação em Tecnologia Ambiental da Universidade de Ribeirão Preto

\section{Ligia Ribeiro da SILVA TONUCI}

Farmacêutica pela Universidade de Ribeirão Preto. Mestre em Tecnologia Ambiental pela Universidade de Ribeirão Preto

\section{Murilo Daniel de MeLlo InNocentini}

Engenheiro Químico pela Universidade Federal de São Carlos. Mestre em Engenharia Química pela Universidade Federal de São Carlos. Doutor em Engenharia Química pela Universidade Federal de São Carlos. Pós-doutorado em Engenharia de Materiais pela Universidade Federal de São Carlos. Docente do Programa de Pós-Graduação em Tecnologia Ambiental da

Universidade de Ribeirão Preto
\end{abstract}

Recebido: 30/10/07 Aceito: 04/06/08

\section{RESUMO}

Resíduos de serviços de saúde da cidade de Ribeirão Preto - SP, previamente esterilizados em autoclave, foram inoculados com microrganismos da espécie $P$. aeruginosa na forma vegetativa para o processamento com microondas em escala de laboratório. Analisou-se a influência do tempo de exposição à radiação $(25,30,35$ e 40 min.) e da potência por unidade de massa de resíduo $(60,80$ e $100 \mathrm{~W} / \mathrm{kg})$ no percentual de inativação dos microrganismos para a umidade de entrada do resíduo de $50 \%$ em base úmida. Os resultados experimentais foram ajustados com base na cinética de primeira ordem para determinação da energia de ativação (no intervalo de 13,9 a $14,1 \mathrm{~kJ} / \mathrm{mol}$ ) e do fator pré-exponencial da lei de Ahrrenius (10,7 $\left.\mathrm{min}^{-1}\right)$ pelo método dos mínimos quadrados. As equaçōes propostas permitiram obter por simulação a constante cinética e estimar o tempo de processamento necessário para fornecer diferentes níveis de inativação. Por exemplo, o tempo de exposição à radiação de $117 \mathrm{~min}$ foi requerido para se obter o nível de inativação 6.Log 10 para os resíduos processados à $100 \mathrm{~W} / \mathrm{kg}$ e à temperatura de $100^{\circ} \mathrm{C}$.

PALAVRAS-CHAVE: Resíduos de serviços de saúde, desinfecção, microondas, Pseudomonas aeruginosa.

\section{INTRODUÇÃO}

A busca por alternativas economicamente viáveis e ambientalmente seguras para o tratamento de resíduos de serviços de saúde (RSS) é um desafio para os responsáveis pelo gerenciamento adequado desses resíduos, sobretudo nos países em desenvolvimento onde os recursos financeiros são escassos. $\mathrm{O}$ gerenciamento dos resíduos de serviços de saúde é de responsabilidade do gerador (Lei 11.445/2007), porém há necessidade de ação integrada entre os órgãos federais, estaduais e municipais de meio ambiente, de saúde e de limpeza urbana com o objetivo de regulamentar o gerenciamento dos resíduos de serviços de saúde (Resolução CONAMA 358/2005).

Public healthcare wastes from the city of Ribeirão Preto-SP, pre-sterilised in an autoclave, were inoculated with microrganisms of the species $P$. aeruginosa in vegetative form for microwave processing on a laboratory scale. An analysis was made of the influence of radiation exposure time $(15,25,30$ and $40 \mathrm{~min})$ and power per waste mass unit (60, 80 and $100 \mathrm{~W} / \mathrm{kg})$ on the percentage of inactivation of the microrganisms at an incoming waste moisture level of $50 \%$ (wet basis). The experimental results were adjusted based on first-order kinetic model. The activation energies (in the range of 13.9 to $14.1 \mathrm{~kJ} / \mathrm{mol}$ ) and the Arrhenius pre-exponential factors $\left(10.7 \mathrm{~min}^{-1}\right)$ were determined by the least squares method. The kinetic parameters obtained allow one to predict by simulation the first-order kinetic constant achieved with P. aeruginosa in typical healthcare waste, based on the radiation exposure time and temperature for some levels of inactivation. For example, the waste disinfection time required for the inactivation level equivalent to $6 . \log 10$ was estimated to 117 min for wastes processed at $100 \mathrm{~W} / \mathrm{kg}$ and at temperatures of $100^{\circ} \mathrm{C}$.

KEYWORDS: Healthcare waste, disinfection, microwave, Pseudomonas aeruginosa. 
de forma a não comprometer o meio ambiente e a saúde pública.

O calor é utilizado em vários processos de tratamento de resíduos. As principais técnicas de tratamento de resíduos de serviços de saúde (RSS) são a desinfecção química, autoclavagem, incineração, pirólises convencional e à plasma, exposição à radiação ionizante e não-ionizante (Diaz, Savage e Eggrth, 2005; Pellerin, 1994).

As microondas não são fontes diretas de calor. $\mathrm{O}$ magnetron gera a onda eletromagnética, com freqüência geralmente de $2450 \mathrm{MHz}$, através do processo de ressonância, a onda é absorvida pelas moléculas de água existentes no resíduo, que proporcionam o aumento do grau de agitação das moléculas, que provoca a dissipação de calor. Conseqüentemente, em razão de sua baixa umidade, os RSS normalmente têm de ser umedecidos com água líquida ou vapor para o tratamento por microondas. Temperaturas da massa de resíduo úmido entre $98^{\circ} \mathrm{C}$ a $105^{\circ} \mathrm{C}$ ocasionariam a destruição dos microrganismos presentes nos resíduos (Diaz, Savage e Eggrth, 2005).

Nos processos de aquecimento são parâmetros importantes a potência da fonte de calor, a massa e a capacidade calorífica da substância ou corpo em aquecimento, o tempo de aquecimento e as características fluidodinâmicas do meio. O equipamento em escala real utilizado para a desinfecção de RSS por microondas é patenteado pela Sanitec Industries em nível mundial (Goldner et al, 1993).

Parece ser consenso na literatura que o processo de inativação microbiana por microondas é predominantemente térmico. Inúmeros trabalhos compararam os resultados obtidos com aquecimento convencional e com microondas e concluíram não haver diferença substancial nos resultados (Goldblith e Wang, 1967; Lechowich et al, 1969; Vela e Wu, 1979; Jeng et al, 1987; Fujikawa, Ushioda e Kudo, 1992). No entanto, todos os ensaios dos trabalhos citados foram realizados em meios líquidos ou mediante condições de dissipação energética (potências por unidade de massa de resíduo de até $4.000 \mathrm{~W} / \mathrm{kg}$ ) que não representam a operação de equipamentos de desinfecção por microondas em escala real.

Tonuci, Paschoalato e Pisani Jr. (2008) mostraram que a inativação de RSS com E. coli por microondas pode não ser efetiva. Utilizaram amostras de RSS de Ribeirão Preto processadas em tempos de exposição de 15 a $40 \mathrm{~min}$, potências de 60 , 80 e $100 \mathrm{~W} / \mathrm{kg}$ para a umidade do resíduo na entrada de $50 \%$ em base úmida. Determinaram os valores de energia de ativação no intervalo de 7.594 a $10.071 \mathrm{~J} / \mathrm{mol} \mathrm{e}$ fator pré-exponencial de $2,13 \mathrm{~min}^{-1}$. Concluíram que, nessas condições, o processo de inativação não é efetivo de forma a atender o nível III de inativação microbiana.

A Resolução ANVISA RDC no 306/2004, que dispõe sobre o Regulamento Técnico para o gerenciamento de RSS, estabelece de forma geral, que os sistemas de tratamento de RSS classes A e E devem ter redução da carga microbiana, em equipamento compatível com Nível III de inativação microbiana, ou seja, inativação de bactérias vegetativas, fungos, vírus lipofílicos e hidrofílicos, parasitas e micobactérias com redução igual ou maior a 6Log 10, e inativação de esporos do $B$. stearothermophilus ou de esporos do $B$. subtilis com redução igual ou maior a $4 \log 10$.

O $P$. aeruginosa tem o formato de bastonete reto, com 0,5 a $0,7 \mu \mathrm{m}$ de diâmetro e 1,5 a 3,0 $\mu \mathrm{m}$ de comprimento. É flagelado, aeróbio, não fermentador de açúcar, com parede celular com poucas camadas de peptideoglicano (Gram-negativo), degrada proteínas, carboidratos, gorduras e substratos afins. No entanto, pode crescer em ambiente anaeróbio em presença de nitrato aceptor final de elétrons. É capaz de crescer em colônias com formação de biofilme e síntese de alginato. Esse agrupamento de indivíduos em microcolônias unidas por uma matriz exopolissacarídea propicia a formação de uma estrutura altamente hidrofóbica que confere resistência à comunidade de laboratório nas seguintes condiçōes:

bactérias, que são altamente resistentes à variação de temperatura de 4 a $42^{\circ} \mathrm{C}$. Esse microrganismo é oportunista, causador de bacteremias severas, de morte em indivíduos debilitados, de infecções urinárias, endocardites, pneumonias, meningites (Lincopan e Trabulsi, 2004).

Monteiro et al (2001) mostraram os custos operacionais das principais técnicas de tratamento de resíduos de serviços de saúde, expressos em dólares americanos por toneladas (Tabela 1), na qual se pode constatar que o custo operacional do processo de inativação por microondas está na faixa intermediária dentre os custos dos demais processos.

$\mathrm{O}$ consumo total de energia na unidade de tratamento por microondas, além da energia dissipada pelos magnetrons, também envolve o consumo pela alimentação do resíduo à moega, trituração em moinho de facas, injeção de vapor saturado para aumento da umidade, transporte do resíduo através da câmara de irradiação e descarregamento do resíduo tratado por rosca sem-fim. $\mathrm{O}$ consumo total de energia é de aproximadamente $270 \mathrm{kWh}$ por tonelada de RSS processada (Epri, 2003).

A carência de dados quantitativos referentes à inativação de microrganismos por microondas motivou a realização do estudo em escala laboratorial, de forma a simular as condiçôes operacionais de um equipamento em escala real.

\section{MATERIAIS E MÉTODOS}

As informações utilizadas na escolha das condições operacionais foram coletadas no aterro sanitário da cidade de Ribeirão Preto-SP, localizado na Rodovia Mário Donegá, $\mathrm{km} 5$. O sistema de desinfecção instalado consiste de 4 a 6 magnetrons, com freqüência de $2450 \mathrm{MHz}$ e $1400 \mathrm{~W}$

Tabela I - Custos operacionais comparativos das principais técnicas de tratamento de RSS em dólares por tonelada (Monteiro et al, 200 I)

\begin{tabular}{cc}
\hline Tipo de processo & Custo operacional (US\$/t) \\
\hline Incineração & 150 a 180 \\
Pirólise & 120 a 180 \\
Radiação ionizante & 75 a 90 \\
Microondas & 75 a 85 \\
Autoclavagem & 45 a 75 \\
Desinfecção química & 35 a 50 \\
\hline
\end{tabular}


de potência cada. Portanto, a potência total do equipamento é de $5600 \mathrm{~W}$ a $8400 \mathrm{~W}$, respectivamente. A capacidade de processamento é de até $250 \mathrm{~kg} / \mathrm{h}$ de resíduos em bateladas de $177 \mathrm{~kg}$ a cada $42,5 \mathrm{~min}$. O tempo de exposição à radiação é de aproximadamente 30 min e a umidade do resíduo na entrada é de $50 \%$ em massa (base úmida). No entanto, o equipamento opera normalmente com a carga de $180 \mathrm{~kg} / \mathrm{h}$, ou seja, $127 \mathrm{~kg}$ em bateladas de 42,5 min com 30 min de exposição à radiação.

Portanto, baseado na potência total do equipamento, na massa de cada batelada e no tempo de exposição à radiação (30 min), foi possível estabelecer as condições operacionais de relação potência por unidade de massa (P/M) no intervalo de 62 a $93 \mathrm{~W} / \mathrm{kg}$, que serviram de referência para estabelecer as condiçôes operacionais de laboratório: Potência em relação à Massa de resíduos $(\mathrm{P} / \mathrm{M})$ de 60, $80 \mathrm{e}$ $100 \mathrm{~W} / \mathrm{kg}$ e tempos de exposição $(\mathrm{t})$ de 25 a 40 minutos.

\section{Fundamentos teóricos}

O processo de desinfecção em batelada associado ao modelo cinético de primeira ordem e à Lei de Arrhenius fornecem a Equação 1 (Geankopolis, 2003):

$\mathrm{N}=\mathrm{N}_{0} . \exp ;-\underset{0}{\# \mathrm{k}_{0}} \cdot \mathrm{e}^{\mathrm{e} \frac{\mathrm{cEa}}{\mathrm{R} . \mathrm{T}} \mathrm{m}} \cdot \mathrm{dt} \mathrm{E}$

na qual $\mathrm{N}$ é o número de microrganismos, $\mathrm{N}_{0}$ é o número de microrganismos no instante inicial, $\mathrm{k}_{0}$ é o fator pré-exponencial $\left(\mathrm{s}^{-1}, \mathrm{~min}^{-1}\right.$ ou h$\left.^{-1}\right)$, Ea é a energia de ativação $(\mathrm{J} / \mathrm{mol})$ e $\mathrm{R}$ é a constante universal dos gases ideais $(8,314 \mathrm{~J} / \mathrm{mol} . \mathrm{K})$ e t é o tempo (s, min ou h).

$\mathrm{O}$ modelo cinético de primeira ordem pressupóe as seguintes hipóteses: população homogênea de microrganismos (cultura pura), escoamento pistonado ou batelada de mistura completa, distribuição homogênea da radiação através do resíduo e temperatura uniforme ao longo do resíduo.

A condição de dispersão uniforme da radiação na cavidade de fornos de microondas domésticos é adequada, sobretudo quando os pratos internos de apoio forem giratórios. As paredes internas dos fornos refletem parte da radiação que resulta em interferência entre ondas em algumas regióes dos fornos. $\mathrm{O}$ movimento giratório minimiza o efeito dessas regiōes no processo de aquecimento (Rosini, Nascentes e Nóbrega, 2004).
Uma vez que os experimentos de desinfecção em batelada ocorreram em condição não-isotérmica em função do tempo, foi preciso levar em consideração a variação de $\mathrm{k}$ ao longo de cada ensaio. A dependência da temperatura em função do tempo pôde ser obtida experimentalmente para cada potência por unidade de massa de resíduo. $\mathrm{O}$ ajuste de um polinômio para representar T em função do tempo permitiu a integração numérica e determinação dos valores de $\mathrm{k}_{0}$ e Ea para as condiçôes estudadas.

O fator $\mathrm{k}_{0}$ é pouco dependente da temperatura e foi assumido como sendo constante no estudo (Levenspiel, 1999). Esse parâmetro deve ser função do tipo de microrganismo e de suas características estruturais. A Ea foi assumida como sendo dependente da intensidade da radiação, por analogia ao efeito de catalisadores em reaçóes químicas, uma vez que é o agente promotor da geração de calor no resíduo.

A integração numérica foi efetuada pelo método de Simpson e a determinação de $\mathrm{Ea}$ e $\mathrm{k}_{0}$ foi realizada pelo critério de mínimos quadrados. $\mathrm{O}$ erro (E) foi determinado para cada intervalo de tempo e foi minimizado pelo critério da somatória dos erros ao quadrado para todas as condições experimentais. A fração de inativação de microrganismos $(\mathrm{X})$ foi definida pela Equação 2 como:

$X(\%)=\frac{\left(\mathrm{N}_{0}-\mathrm{N}\right)}{\mathrm{N}_{0}}$

\section{Equipamentos e acessórios}

$\mathrm{Na}$ realização dos experimentos foi utilizado um forno de microondas doméstico, da marca SHARP, modelo R-4B82, com freqüência de $2450 \mathrm{MHz}$ e volume interno de $20 \mathrm{~L}$. Os acessórios e materiais utilizados foram: termômetro digital, balança digital, placa de Petri de $90 \mathrm{~mm}$, alça bacteriológica descartável calibrada de $10 \mu \mathrm{L}$, autoclave, estufa bacteriológica com temperatura controlada entre 35 e $37^{\circ} \mathrm{C}$, higrômetro Sartorius modelo MA 30, capela de fluxo laminar, cepas do tipo padrão de P. aeruginosa derivada ATCC® 27853, meio de cultura líquido BHI, meio de cultura Manitol e soro fisiológico 0,9\% em massa.

O volume total de RSS coletado para compor as amostras foi de aproximadamente $200 \mathrm{~L}$. As coletas foram realizadas em 3 dias distintos (de terça á quinta-feira) após o processamento na unidade de microondas em escala real instalada junto ao aterro sanitário de Ribeirão Preto, porém antes de ser aterrado. Para eliminar os riscos de manipulação do material, foi realizada a autoclavagem desse volume de resíduos para esterilização com vapor à 2,0 atm (absoluta) durante 15 minutos. O método do quarteamento descrito na NBR 10.007/2004 foi utilizado para gerar uma amostra representativa do volume inicial com aproximadamente $2,0 \mathrm{~kg}$ do resíduo para separação por catação e determinaçãa da composição gravimétrica do resíduo utilizado no estudo (Tabela 2).

\section{Procedimentos experimentais}

O procedimento experimental do estudo envolveu as etapas de calibração da potência dissipada pelo forno, determinação da umidade da massa de resíduos, preparação dos microrganismos, ensaios de desinfecção, recuperação dos microrganismos por imersão do resíduo em solução salina (Tonuci, Paschoalato e Pisani Jr., 2008).

Tabela 2 Composição gravimétrica do RSS utilizado

\begin{tabular}{cc}
\hline Materiais & $\%$ em massa \\
\hline Plástico fino & 46,24 \\
Tecido/papel & 24,32 \\
Plástico duro & 22,47 \\
Látex & 4,26 \\
Vidro & 1,92 \\
Alumínio & 0,76 \\
Outros (algodão, madeira e material pulverulento) & 0,03 \\
\hline
\end{tabular}




\section{Calibração do forno de microondas}

A calibração da potência do forno de microondas foi realizada em $10 \%$, $20 \%$ e $30 \%$ da potência nominal do forno, conforme a indicação no aparelho. Inicialmente, colocava-se $700 \mathrm{~g}$ de água deionizada em um béquer de $1000 \mathrm{~mL}$. Media-se a temperatura inicial da água e posicionava-se o béquer no centro da cavidade do forno de microondas, após programá-lo para 120 segundos na potência desejada. Ao término, removia-se béquer e media-se novamente a temperatura da água.

$\mathrm{O}$ experimento foi realizado em triplicata para obtenção da potência real dissipada, que foi calculada pelo princípio da conservação da energia. A Equação 3 foi utilizada para calcular a potência real dissipada:

$P=\frac{M_{A} \cdot C_{\text {PA }} \cdot D T}{t}+\frac{M_{v} \cdot C_{p v} \cdot D T}{t}$

em que $\mathrm{P}$ é a potência real absorvida pela amostra (W), $M_{A}$ é a massa da amostra de água $(\mathrm{kg}), \mathrm{C}_{\mathrm{PA}}$ é a capacidade calorífica ou calor específico da água (J/kg.K), $\mathrm{C}_{\mathrm{PV}}$ é a capacidade calorífica ou calor específico do vidro (J/kg.K), t é o tempo (s) e $\Delta \mathrm{T}$ é a diferença de temperatura (K). Por hipótese, assumiu-se que a temperatura do vidro era igual a da água em cada ensaio de calibração.

\section{Determinação da umidade do resíduo de serviço de saúde}

Determinou-se a umidade do resíduo com o higrômetro Sartorius MA 30 com os seguintes parâmetros: temperatura de $105^{\circ} \mathrm{C}$, tempo de $99 \mathrm{~s}$ e umidade de 0 a $105 \%$.

Foram recolhidas amostras do RSS na unidade de desinfecção por microondas junto ao aterro sanitário de Ribeirão Preto, antes do início da exposição às microondas, logo após trituração e umidificação.

A umidade do RSS foi medida antes e depois de cada experimento realizado em laboratório, ou seja, no início e no final dos ensaios de contaminação do RSS para a exposição às microondas. A equação 4 foi utilizada para o cálculo da quantidade de água a ser adicionada ao resíduo para se manter a umidade inicial do experimento constante e igual ao valor encontrado na entrada do equipamento em escala real.
$U=\frac{M_{\mathrm{H}_{2} \mathrm{O}_{\text {incial }}}+\mathrm{M}_{\mathrm{H}_{2} \mathrm{O}_{\text {adicionala }}}}{\mathrm{M}_{\mathrm{H}_{2} \mathrm{O}_{\text {inicial }}}+\mathrm{M}_{\mathrm{H}_{2} \mathrm{O}_{\text {adicionata }}}+\mathrm{M}_{\mathrm{RSS} \text { seco }}}$

nas quais, $\mathrm{M}$ é a massa $(\mathrm{kg})$ e $\mathrm{U}$ é a umidade em base úmida. A quantidade de água adicionada foi calculada para manutenção da umidade do resíduo constante em 50\% (base úmida), valor encontrado na operação da umidade em escala real.

\section{Preparação do microrganismo}

O microrganismo utilizado foi Pseudomonas aeruginosa cepa derivada ATCC® 27853. A preparação consistiu em adicionar $0,2 \mathrm{~mL}$ de água destilada para reidratar a amostra por 15 minutos. Em seguida, transferiu-se o conteúdo para um tubo de ensaio contendo o meio de cultura líquido BHI (Brain - Heart - Infusion), caldo infuso de cérebro e coração. A partir da cultura de células crescidas no caldo, foram alicotados $10 \mu \mathrm{L}$ da suspensão com uma alça plástica calibrada. Esse volume foi disperso em $50 \mathrm{~mL}$ de solução salina $0,9 \%$ em massa, previamente esterilizada. Nesta etapa, foi retirada uma amostra de $10 \mu \mathrm{L}$ que foi semeada em placas de Petri com meio sólido de cultura sólido Manitol; em oito direçōes para o cálculo da quantidade de microrganismo inoculada no resíduo no início de cada experimento $\left(\mathrm{N}_{0}\right)$. Incubadas as placas por $24 \mathrm{~h}$ em estufa, com temperatura controlada de 36 a $37^{\circ} \mathrm{C}$, as unidades formadoras de colônia (UFC) foram contadas.

\section{Ensaio de desinfecção}

Primeiramente, ajustava-se o forno de laboratório para dissipar 10\% de sua capacidade nominal, potência real de 66 W. Em seguida, media-se a umidade do resíduo e calculava-se a massa de água a ser adicionada para fornecer a umidade de 50\% (em base úmida). As massas de resíduos úmidos utilizadas para fornecer as potências por unidade de massa de 60,80 e $100 \mathrm{~W} / \mathrm{kg}$ foram respectivamente $1095 \mathrm{~g}, 821 \mathrm{~g}$ e $657 \mathrm{~g}$ (massas de resíduos úmidos). Inoculava-se o resíduo com $50 \mathrm{~mL}$ de solução salina $0,9 \%$ contendo nela $10 \mu \mathrm{L}$ da suspensão com microrganismos e completava-se a massa de água restante. Ajustava-se o equipamento para o tempo de operaçáo necessário $(25,30,35$ e $40 \mathrm{~min})$.
O mesmo procedimento foi repetido, porém sem a adição de microrganismos, para se determinar a temperatura do resíduo para os tempos de operação estudados, uma vez que a constante de velocidade do processo de desinfecção é dependente da temperatura, de acordo com a lei de Arrhenius. A temperatura foi assumida uniforme em cada instante no interior da massa de resíduos, uma vez que a espessura de sua camada dentro do forno foi de aproximadamente $5,0 \mathrm{~cm}$. A temperatura foi medida no centro da massa de resíduos e admitida representativa do todo para cada instante.

\section{Recuperação dos microrganismos por imersão do RSS em solução salina $0,9 \%$}

O teste para a recuperação dos microrganismos foi feito mediante a retirada de $10 \mu \mathrm{L}$ com alça plástica calibrada da suspensão preparada com microorganismo, esse volume foi disperso em $50 \mathrm{~mL}$ solução salina $0,9 \%$ esterilizada, recolhido $10 \mu \mathrm{L}$ deste e semeado em placas de Petri com meio sólido de cultura para o microrganismo em oito direçôes. Esse procedimento permitiu quantificar o número de microrganismos inoculado no RSS. Calculada a quantidade de água necessária para alcançar umidade do RSS em $50 \%$ (base úmida), foi contaminada a massa conhecida de RSS, porém sem exposição à radiação, o resíduo foi totalmente imerso em $4 \mathrm{~L}$ de solução salina $0,9 \%$ esterilizada e homogeneizado por 4 minutos. Foram recolhidas 4 amostras de $1 \mathrm{~mL}$ com pipeta de Pasteur, semeadas em placas de Petri com meio de cultura seletivo, incubadas por $24 \mathrm{~h}$ em estufa com temperatura controlada $36-37^{\circ} \mathrm{C}$. Posteriormente, foi efetuada a contagem de unidades formadoras de colônia que permitiu a comparação com a quantidade de microrganismo que foi inoculada no RSS. Esse procedimento teve como objetivo validar a metodologia utilizada para a realização da pesquisa para se determinar as quantidades de microrganismos inicial e final em cada ensaio, $\mathrm{N}_{0}$ e $\mathrm{N}$ respectivamente (Tonuci, 2006).

A massa total de resíduos com $50 \%$ de umidade em base úmida utilizada nessa etapa foi de $1095 \mathrm{~g}$, que continha $174,31 \mathrm{~g}$ de água antes do umedecimento. A quantidade de água 
necessária para resultar na umidade de $50 \%$ em base úmida foi então de 373,19 g. A adição de $4000 \mathrm{~mL}$ de solução salina para submergir o resíduo totalizou em $4535,5 \mathrm{~g}$ de água e no volume de água de $4549 \mathrm{~mL}$. O total de unidades formadoras de colônias nas 4 placas foi 9 . O cálculo de diluição resultou no total de unidades formadoras de colônias recuperadas da amostra de 10235. A quantidade inicialmente inoculada foi de $10^{4}$ bactérias. Portanto, o desvio percentual obtido foi e aproximadamente $2 \%$. Esse resultado possibilitou verificar que o procedimento de recuperação dos microrganismos foi adequado (Tonuci, Paschoalato e Pisani Jr., 2008).

\section{RESULTADOS E DISCUSSÕES}

$\mathrm{Na}$ desinfecção de RSS, é preciso estimar o tempo necessário para se obter a inativação desejada. Os ensaios de desinfecção realizados em batelada, ou a existência de perfis térmicos em equipamentos contínuos, resultam em uma dependência da temperatura do resíduo em função do tempo, ou da posição. Portanto, é necessário explicitar a temperatura em função do tempo para cada uma das potências para se determinar os parâmetros $\mathrm{k}_{0}$ e Ea pelo método dos mínimos quadrados.

A Figura 1 mostra a temperatura do resíduo em função do tempo e da potência.

$\mathrm{O}$ ajuste dos pontos experimentais através de uma função polinomial do segundo grau se mostrou satisfatório para o intervalo estudado, pois resultou em coeficientes de correlação próximos a 1,0. É importante destacar que as funções ajustadas não podem ser extrapoladas, pois podem resultar em temperaturas decrescentes em função do tempo quando os pontos de máximo das funçôes forem suplantados.

A Tabela 3 e a Figura 2 mostram os resultados experimentais e calculados obtidos para as condições estudadas.

Nota-se pela Figura 2 que o modelo de primeira ordem superestimou a fração de inativação quando comparado com os resultados experimentais. $\mathrm{O}$ modelo de Chick (cinética de $1^{\mathrm{a}}$ ordem) leva em consideração apenas o efeito térmico na inativação dos microrganismos. Os desvios percentuais obtidos estiveram na faixa de 3 a $12 \%$ para $60 \mathrm{~W} / \mathrm{kg}$, de 2 a $14 \%$ para $80 \mathrm{~W} / \mathrm{kg}$ e de

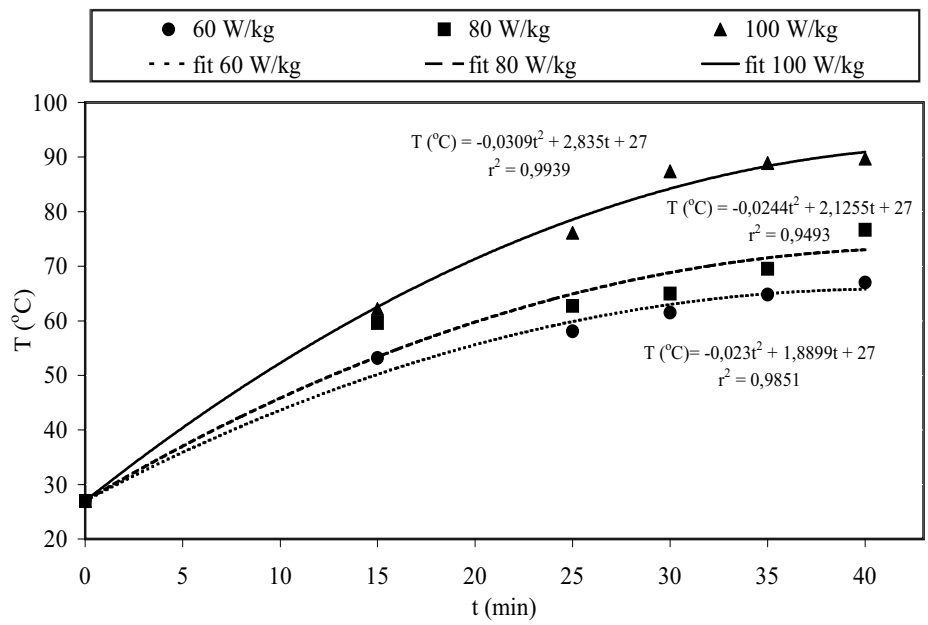

Figura I - Temperatura do resíduo em função do t e da P/M para $50 \%$ de umidade (base úmida)

Tabela 3 - Número de microrganismos e fração de inativação experimentais

\begin{tabular}{ccccc}
\hline $\begin{array}{c}\mathrm{P} / \mathrm{M} \\
(\mathrm{W} / \mathrm{kg})\end{array}$ & $\begin{array}{c}\mathrm{t} \\
(\mathrm{min})\end{array}$ & $\begin{array}{c}\mathrm{N}_{0} \\
(\mathrm{UFC})\end{array}$ & $\begin{array}{c}\mathrm{N} \\
(\mathrm{UFC})\end{array}$ & $\begin{array}{c}\mathrm{X} \\
(\%)\end{array}$ \\
\hline 60 & 25 & 26600400 & 6805700 & 74 \\
& 30 & 73890000 & 10560490 & 86 \\
& 35 & 50902000 & 5427000 & 89 \\
80 & 40 & 7183500 & 571356 & 92 \\
& 25 & 1980000 & 429794 & 78 \\
& 30 & 10800000 & 2051139 & 81 \\
& 35 & 10800000 & 1945140 & 82 \\
& 40 & 1980000 & 117790 & 94 \\
& 25 & 2180000 & 434644 & 80 \\
& 30 & 10800000 & 1402294 & 87 \\
& 35 & 1980000 & 158276 & 92 \\
& 40 & 10800000 & 81117 & 99 \\
\hline
\end{tabular}

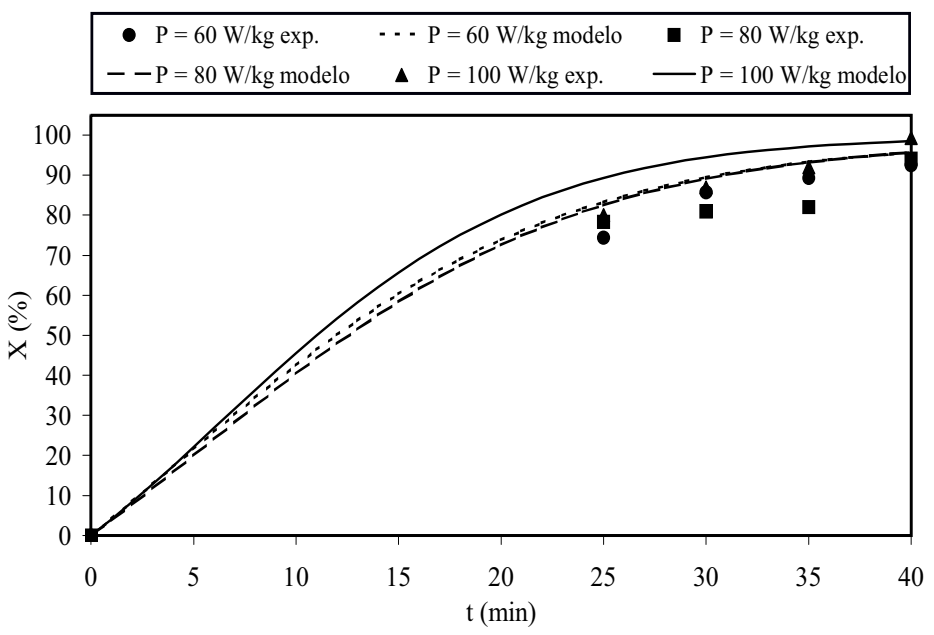

Figura 2 - Fração de inativação da P. aeruginosa para umidade de $50 \%$ 
1 a $12 \%$ para $100 \mathrm{~W} / \mathrm{kg}$. Valores superiores ao obtido na validação da técnica de recuperação dos microorganismos por imersão em solução salina $(2 \%)$.

É sabido que o microorganismo $P$. aeruginosa é capaz de se desenvolver na forma de biofilmes nas superfícies dos materiais presentes no resíduo. Esse processo pode ocorrer no intervalo de horas ou minutos. Portanto, é possível que tenha ocorrido a formação camadas sobre as superfícies das partículas e que estas tenham dificultado a propagação do calor até os níveis necessários para ocorrer a inativação do microrganismo (O'toole e Kolter, 1998). Por outro lado, o alginato, por ser altamente hidrofóbico, pode ter repelido a água da superfície das colônias, e assim, é possível que a umidade dos pontos em que estiveram localizados os microrganismos tenha sido inferior a do restante da massa de resíduo e portanto, tenham sofrido menor aquecimento, resultando em frações de inativação inferiores ao previsto pelo modelo.

As energias de ativação resultantes foram: $13855 \mathrm{~J} / \mathrm{mol}$ para $60 \mathrm{~W} / \mathrm{kg}, 14136 \mathrm{~J} / \mathrm{mol}$ para $80 \mathrm{~W} / \mathrm{kg}$ e $13980 \mathrm{~J} / \mathrm{mol}$ para $100 \mathrm{~W} / \mathrm{kg}$. Portanto, as equações 5 a 7 podem ser utilizadas para o cálculo da constante de velocidade nas condiçôes estudadas.

para $60 \mathrm{~W} / \mathrm{kg}$

$\mathrm{k}=10,7 \mathrm{~min}^{-1} \cdot \operatorname{expc} \frac{-13855 \mathrm{~J} / \mathrm{mol}}{\mathrm{RT}} \mathrm{n}$

para $80 \mathrm{~W} / \mathrm{kg}$

$\mathrm{k}=10,7 \mathrm{~min}^{-1} \cdot \operatorname{expc} \frac{-14136 \mathrm{~J} / \mathrm{mol}}{\mathrm{RT}} \mathrm{n}$

para $100 \mathrm{~W} / \mathrm{kg}$

$\mathrm{k}=10,7 \mathrm{~min}^{-1} \cdot \exp \mathrm{c} \frac{-13980 \mathrm{~J} / \mathrm{mol}}{\mathrm{RT}} \mathrm{n}$

Os valores obtidos foram praticamente independentes da potência aplicada. Nota-se pela Figura 2 que o aumento da fração de inativação do $P$. aeruginosa é provocado preponderantemente pelo aumento da temperatura da massa de resíduos. O efeito do aumento na constante de velocidade não ocorre em função da redução da energia de ativação.

As equações de previsão da constante cinética na forma da Lei de Arrhenius permitem avaliar a técnica de tratamento através da previsão do tempo de inativação necessário para se obter os níveis de desinfecção 4.Log 10 e 6.Log 10. A literatura cita que a tem- peratura do processo em escala real é de aproximadamente $100^{\circ} \mathrm{C}$. A simulação do processo de desinfecção à temperatura constante com a cinética de primeira ordem permite estimar os tempos necessários para se obter os níveis de inativação desejados. A Tabela 4 mostra os resultados obtidos para essa simulação.

Verifica-se pela Tabela 4 que mesmo para a temperatura de $100^{\circ} \mathrm{C}$ os tempos calculados são bastante superiores ao tempo de exposição praticado no equipamento em escala real (30 min). Isso sugere que a técnica de inativação não é efetiva nas condições operacionais praticadas na unidade em escala real (tempo de exposição à radiação de 30 min e P/M de 62 a $93 \mathrm{~W} / \mathrm{kg}$ ).

Caso o equipamento operasse de forma a garantir o nível de inativação 6.Log 10 para o microrganismo P. aeruginosa, ou seja, a $100 \mathrm{~W} / \mathrm{kg}$ e com tempo de exposição à radiação de $117 \mathrm{~min}$, foi possível estimar o custo energético devido à etapa de tratamento na câmara de irradiação. Segundo à ANEEL (2007), o preço médio da energia elétrica para uso industrial no Brasil foi de $\mathrm{R} \$ 216,61$ por MWh. Sendo assim, o custo energético da etapa de inativação seria de R\$ 42,24 por tonelada de RSS tratado (US\$ 24,14 para câmbio atual de $\mathrm{R} \$ 1,75$ para cada dólar). Já o custo operacional total do processo com base no consumo de $270 \mathrm{kWh}$ por tonelada de RS, o valor seria de $\mathrm{R} \$ 58,48$ por tonelada de RSS (US\$ 33,42 para câmbio de $\mathrm{R} \$ 1,75$ para cada dólar). Esse valor é consideravelmente menor que o explicitado na Tabela 1 para inativação por microondas e compatível com os custos operacionais das técnicas mais econômicas de autoclavagem e desinfecção química.

Tabela 4 - Resultados do modelo de primeira ordem para a previsão do tempo de inativação do $P$. aeruginosa equivalente aos níveis de inativação 4. $\log 10$ e 6. $\log 10$ a $100^{\circ} \mathrm{C}$

\begin{tabular}{cccc}
\hline Nível de inativação & $\begin{array}{c}\mathrm{P} \\
(\mathrm{W} / \mathrm{kg})\end{array}$ & $\begin{array}{c}\mathrm{k} \\
\left(\mathrm{min}^{-1}\right)\end{array}$ & $\begin{array}{c}\mathrm{t} \\
(\mathrm{min})\end{array}$ \\
\hline 4.Log 10 & 60 & 0,123 & 75 \\
6.Log 10 & 60 & 0,123 & 113 \\
4.Log 10 & 80 & 0,112 & 82 \\
6.Log 10 & 80 & 0,112 & 123 \\
4.Log 10 & 100 & 0,118 & 78 \\
6.Log 10 & 100 & 0,118 & 117 \\
\hline
\end{tabular}

\section{CONCLUSÕES}

A fração de inativação se mostrou dependente do tempo e da potência por unidade de massa dos resíduos durante o tratamento. A máxima fração de inativação experimental de $99 \%$ foi obtida para a potência de $100 \mathrm{~W} / \mathrm{kg}$ e $40 \mathrm{~min}$ de exposição.

Os resultados obtidos para $\mathrm{Ea}$ e $\mathrm{k}_{0}$ foram consistentes, pois, de maneira geral, os desvios percentuais entre os valores experimentais e calculados pelo modelo cinético de primeira ordem foram aceitáveis, e o modelo foi capaz de descrever o comportamento dos pontos experimentais, no entanto superestimou os valores da fração de inativação quando comparados com os resultados experimentais.

$\mathrm{O}$ aumento de $\mathrm{X}$ foi provocado preponderantemente pelo aumento da temperatura da massa de resíduos. $\mathrm{O}$ efeito do aumento da constante de velocidade não ocorreu em função da redução da energia de ativação. Esses dois efeitos permitem atribuir uma baixa sensibilidade do microorganismo ao processo de desinfecção por microondas. $\mathrm{O}$ tempo de exposição à radiação se mostrou mais influente na fração de inativação que a potência por unidade de massa de resíduo nas condições estudadas.

Os tempos de desinfecção necessários para os níveis de inativação equivalentes a $4 \log 10$ e $6 \log 10$, estimados entre 75 e $123 \mathrm{~min}$, denotam que a técnica de inativação de RSS pode ser efetiva, desde que as condições operacionais sejam bem estabelecidas, este não parece ser o caso das condiçôes operacionais do equipamento em escala real tomado como base para o estudo. 


\section{REFERÊNCIAS}

ABNT - ASSSOCIAÇ̃̃O BRASILEIRA DE NORMAS TÉCNICAS. NBR 10007: Amostragem de resíduos sólidos. Rio de Janeiro, 2004.

AGÊNCIA NACIONAL DE ENERGIA ELÉTRICA. Tarifas - Consumidores Finais. In: Agência Nacional de Energia Elétrica, Brasília. Disponível em http://www.aneel.gov. br/area.cfm?idArea $=91 \&$ idPerfil $=2$. Acesso em $14 / 04 / 2008$.

BRASIL. Conselho Nacional do Meio Ambiente. Resolução no 358, de 29 de abril de 2005. Dispōe sobre o tratamento e a disposição final dos resíduos dos serviços de saúde e dá outras providências. Brasília, DF, 2005.

BRASIL. Agência Nacional de Vigilância Sanitária. Resolução RDC no 306, de 7 de dezembro de 2004. Dispóe sobre o Regulamento Técnico para o gerenciamento de resíduos de serviços de saúde. Brasília, DF, 2004.

BRASIL. Congresso Nacional. Lei Federal $n^{o}$ 11.445, de 5 de Janeiro de 2007. Estabelece diretrizes nacionais para o saneamento básico; altera as Leis nos 6.766, de 19 de dezembro de 1979 8.036, de 11 de maio de 1990, 8.666, de 21 de junho de 1993, 8.987, de 13 de fevereiro de 1995; revoga a Lei no 6.528, de 11 de maio de 1978; e dá outras providências.

DIAZ, L. et al. Alternatives for treatment and disposal of healthcare wastes in developing countries. Waste Management, n. 25, p. 626-637, 2005.

ELECTRIC POWER RESEARCH INSTITUTE Emerging Environmental Technologies: An Analysis of New Treatment Technologies for the California Energy Commission. California Energy Commission, Palo Alto, CA, USA, 106p., 2003.
FUJIKAWA, H., USHIODA, H., KUDO, Y. Kinetics of Escherchia coli destruction by microwave irradiation. Applied and Environmental Microbiology, n. 58, p. 920-924, 1992.

GEANKOPLIS, C. J. Transport Processes and Separation Process Principle. 4 ed. Prentice Hall, Englewood Cliffs, NJ, USA, 1025p.,2003.

GOLDBLITH, S. A., WANG, D. I. C. Effect of microwaves on Escherichia coli and Bacillus subtilis. Applied Microbiology, n. 15, p. 1371-1375, 1967.

GOLDNER, H., KAMANN, R., LEINSKI, H. Apparatus and process for treating medical hazardous wastes. US Patent no 5270000 , December, 1993.

JENG, D. K. H.et al. Mechanism of microwave sterilization in the dry state. Applied and Environmental Microbiology, n. 53, p. 2133-2137, 1987.

LECHOWICH, R. V. et al. A procedureforevaluating the effects of 2,450-Megahertz microwaves upon Streptococcus faecalis and Saccharomyces cerevisiae. AppliedMicrobiology, n. 17, p. 106-110, 1969.

LEVENSPIEL, O. Chemical Reactor Engineering. 3 ed. John Wiley and Sons, New York, NY, USA, 668p., 1999.

LINCOPAN, N., TRABULSI, L. R. Pseudomonas aeruginosa. In: TRABULSI, L. R., ALTERTHUM, F. Microbiologia. 4 ed. São Paulo: Editora Atheneu, p. 359-375. 2004.

MONTEIRO, J. H. P. et al. Manual de Gerenciamento Integrado de Resíduos Sólidos. Instituto Brasileiro de Administração Municipal, Rio de Janeiro, RJ, Brasil, 193p., 2001.

O'TOOLE, G. A., KOLTER, R. Flagellar and twitching motility are necessary for Pseudomonas aeruginosa biofilm development. Molecular Microbiology, n. 30, p. 295-304, 1998.

PELLERIN, C. Alternatives to incineration: There's more than one way to remediate. Environmental Health Perspectives, n. 12, p. 840-845, 1994.

ROSINI, F., NASCENTES, C. C., NÓBREGA, J. A. Didactic experiments involving microwave radiation. Química Nova, n. 27, p. 1012-1015, 2004.

TONUCI, L. R. S. Desinfecção de Resíduos de Serviços de Saúde por Aquecimento Dielétrico do Tipo Microondas. Ribeirão Preto, 2006. Dissertação de mestrado-Programa de Pós-graduação em Tecnologia Ambiental-Universidade de Ribeirão Preto, 108p., 2006.

TONUCI, L. R. S., PASCHOALATO, C.F.P.R., PISANI JR, R. Microwave inactivation of Escherichia coli in healthcare waste. Waste Management, n. 28, p. 840-848, 2008.

VELA, G. R., WU, J. F. Mechanism of lethal action of 2, 450-MHz radiation on microorganisms. Applied and Environmental Microbiology, n. 37, p. $550-553,1979$

\section{Endereço para correspondência:}

Reinaldo Pisani Júnior Avenida Costábile Romano, $n^{\circ}$ 2201 Ribeirânia I4096-900 Ribeirão Preto - SP - Brasil Tel: (16) 36036784 E-mail: pisanijr@terra.com.br 